\title{
Exclusive Processes in Diffraction at HERA
}

\author{
A. Polini (on behalf of the H1 and ZEUS Collaborations) \\ INFN Bologna, via Irnerio 46, 40126 Bologna, Italy
}

Received on 11 January, 2007; revised version received on 21 March, 2007

\begin{abstract}
Recent results from the $\mathrm{H} 1$ and ZEUS experiments on exclusive processes in diffractive $e p$ collisions at HERA are reviewed. The measurements discussed here include dijets and charm in photoproduction as well as in deep inelastic scattering. Such processes are sensitive to the partonic structure of the diffractive exchange, particularly to the gluon content, and represent complementary tests for the validity of QCD factorization in diffraction. The measurements are compared to theoretical models, including NLO predictions, using the most recent diffractive parton densities as extracted from inclusive measurements. Recent results on exclusive vector meson production and deeply virtual compton scattering are also presented.
\end{abstract}

Keywords: Diffraction; Charm; Deep inelastic scattering; Vector meson; DVCS

\section{INTRODUCTION}

In diffractive $e^{ \pm} p$ processes at HERA, a photon of virtuality $Q^{2}$ from the incoming lepton interacts with a colour-singlet object within the proton, usually identified with the Pomeron. The final-state signature for such events is characterized by the presence of a large rapidity gap with no hadronic energy flow along the direction of the outgoing proton. The QCD factorization theorem[1] predicts that the cross sections for diffractive Deep Inelastic Scattering (DIS, $Q^{2}>1 \mathrm{GeV}^{2}$ ) can be factorized into a set of universal diffractive Parton Density Functions (dPDFs) convoluted with process-dependent hard scattering coefficients, which can be calculated within perturbative QCD. Of particular interest are the measurements of open charm and of dijet photoproduction $\left(Q^{2} \sim 0\right)$ which provide a direct probe of the gluon content of the Pomeron, and allow to perform complementary tests of the QCD factorization thanks to the presence of well-identified hard scales.

\section{DIFFRACTIVE DIJET PRODUCTION}

Diffractive dijet cross sections have been measured by $\mathrm{H} 1$ and ZEUS in DIS [2, 3] and photoproduction [2, 4].

Figure 1 shows the ZEUS measurement based on $65 \mathrm{pb}^{-1}$ of DIS data $\left(5<Q^{2}<100 \mathrm{GeV}^{2}\right)$ with $\gamma^{*} p$ center-of-mass energy in the range $100<W<250 \mathrm{GeV}$ and the jets reconstructed using the $k_{T}$ algorithm in the $\gamma^{*} p$ center-of-mass system. The cross sections are compared to predictions from Next-to-Leading Order (NLO) QCD calculations using dPDFs extracted from inclusive diffractive DIS data $[5,6]$. The good agreement observed in both shape and normalization between data and predictions supports QCD factorization in DIS.

In photoproduction both $\mathrm{H} 1$ and ZEUS have observed the rate of dijet production uniformly suppressed by a factor of about 0.5 compared to the predictions. This is reminiscent of the observed breakdown of factorization in diffractive dijet production in $p \bar{p}$ collisions at the Tevatron [7]. Fig. 2 shows the distributions of $z_{\mathbb{P}}$ and $x_{\gamma}$, the reconstructed fraction of the momentum carried by the partons of the exchanged Pomeron and the photon, respectively, participating to the hard scatter-

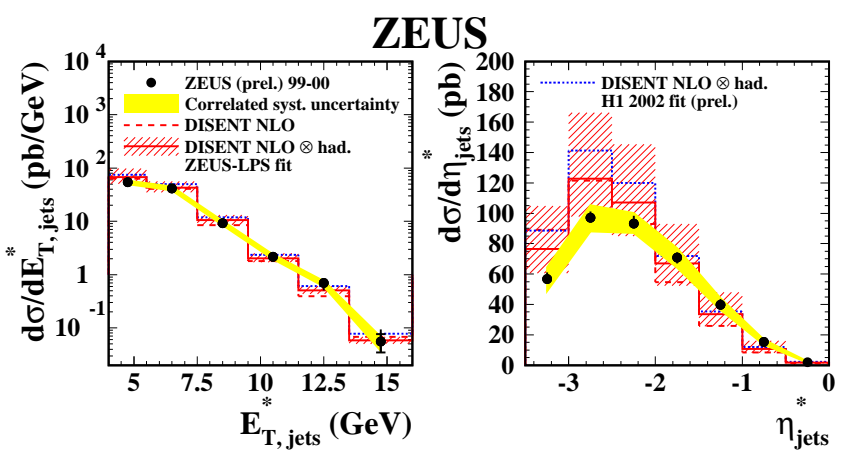

FIG. 1: Comparison of the measured differential cross sections of dijets in diffractive DIS as function of the jet transverse energy $E_{T}^{*}$, and the jet preudorapidity $\eta$ with three different NLO calculations available. The inner (outer) error bands of the points show the statistical only (total) errors. The PDF uncertainties are not displayed.

\section{H1 Diffractive $\gamma p$ Dijets}

- H1 Preliminary H12002 fit (prel.) correl. uncert. $\quad$ FR NLO* $\left(1+\delta_{\text {had }}\right)$

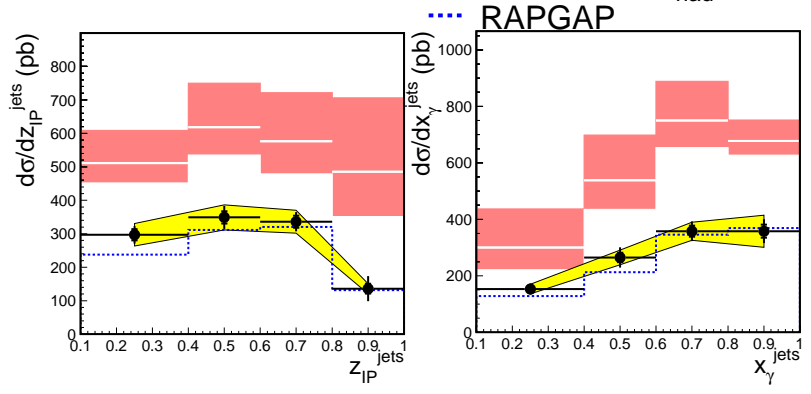

FIG. 2: Comparison of the measured differential cross sections of dijet in diffractive photoproduction as function of $z_{\mathbb{P}}, x_{\gamma}$ with different NLO calculations. The inner (outer) error bands of the points show the statistical only (total) errors.

ing. The fact that the data and the NLO calculations are similar in shape over the full range of $x_{\gamma}$ but differ in normalization disfavours the interpretation of the breaking of factorization as the effect of secondary interactions among the spectator partons, which are more abundantly present in low- $x_{\gamma}$ events. 


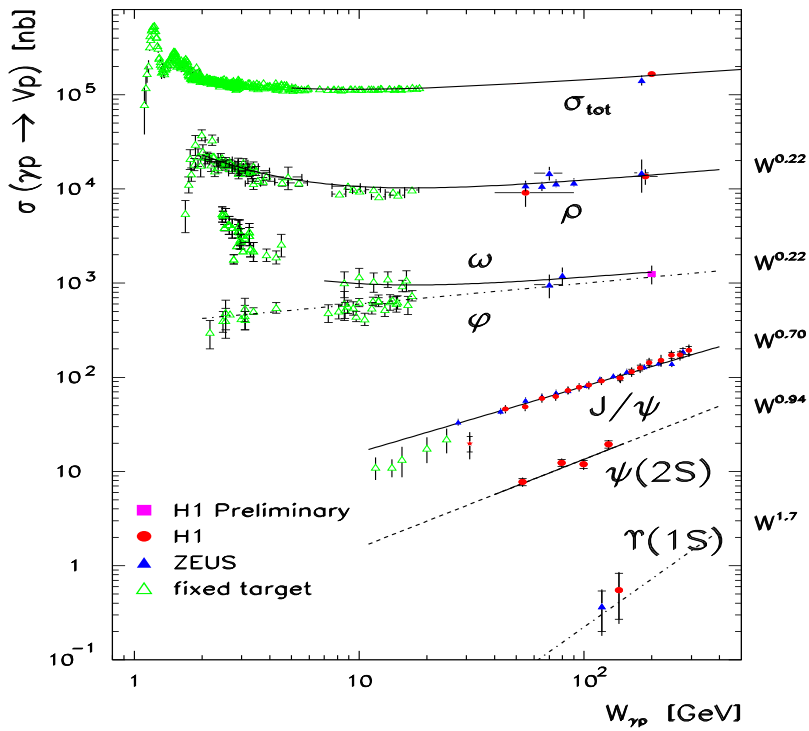

FIG. 3: Exclusive cross section as a function of the $\gamma^{*} p$ center-ofmass energy for various vector mesons in photoproduction together with the total photoproduction cross section. The transitions in the slope from soft to hard scattering is clearly visible when moving from $\gamma p$ scattering to light and heavy vector mesons production.

\section{DIFFRACTIVE CHARM PRODUCTION}

H1 has recently published measurements [8] of diffractive open charm production in DIS and photoproduction using an integrated luminosity of $42.6 \mathrm{pb}^{-1}$. Two independent methods of charm reconstruction were used to extract the diffractive charm cross section. In the first, the charm quark is tagged by the reconstruction of a $D^{*}(2010)$ meson. This technique is used in DIS and photoproduction. In the second, a method based on the displacement of tracks from the primary vertex is used to measure the open charm contribution to the inclusive diffractive cross section in DIS. A good agreement with the $D^{*}$ measurement by ZEUS [9] and with NLO QCD calculations is observed in the full kinematic regime, in favour of the validity of QCD factorization for open charm production both in diffractive DIS and photoproduction.

More precise mesurements and theoretical efforts are necessary to clarify the discrepancy observed between dijets and charm in photoproduction.

\section{EXCLUSIVE AND DIFFRATIVE VECTOR-MESON PRODUCTION}

The dynamics of diffractive interactions has also been extensively studied through exclusive vector meson production $e^{ \pm}+p \longrightarrow e^{ \pm}+V+Y$, where $V=\rho^{0}, \omega, J / \psi, \ldots$ and $Y$ is either an elastically scattered proton or a low-mass state dissociative system. Fig. 3 shows a collection of previous measurements of the exclusive vector meson production cross sections by $\mathrm{H} 1$ and ZEUS and fixed target experiments as a function of the center-of-mass energy $\mathrm{W}$ of the $\gamma^{*} p$ system. The photoproduction of the light vector mesons $\left(\rho^{0}, \omega\right.$ and $\left.\phi\right)$ is character-
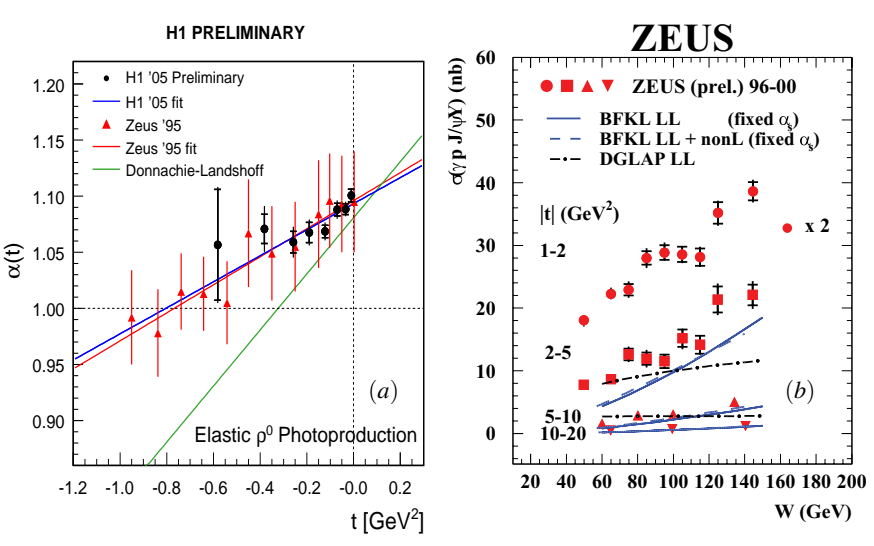

FIG. 4: (a) The measurement by $\mathrm{H} 1$ of $\alpha_{\mathbb{P}}(t)=1.093 \pm$ $0.003_{-0.007}^{+0.008}+\left(0.116 \pm 0.027_{-0.046}^{+0.036} \mathrm{GeV}^{-2}\right) \cdot t$ for elastic $\rho^{0}$ photoproduction. (b) The $J / \psi$ cross section as function of $W$ as measured by ZEUS in four different $t$ bins. DGLAP and BFKL calculations are superimposed.

ized by a soft dependence of the cross sections as a function of $W$. This can be interpreted in the framework of Regge theory as due to the exchange of a Pomeron $(\mathbb{P})$ resulting in an energy dependence of the form $\mathrm{d} \sigma / \mathrm{dt} \propto \mathrm{W}^{4\left(\alpha_{\mathbf{P}}(\mathrm{t})-1\right)}$, and $\alpha_{\mathbb{P}}(t)$ similar to the soft pomeron model $\alpha_{\mathbb{P}}(t)=\alpha_{\mathbb{P}}(0)+\alpha^{\prime} t \simeq$ $1.08+0.25 t$, where $t$ is the square of the momentum transfer at the proton vertex.

Using a sample of more than 240000 events taken in 2005 , H1 has performed a new measurement [10] of exclusive $\rho^{0}$ photoproduction in the kinematic range $20<W<90 \mathrm{GeV}$ and $|t|<3 \mathrm{GeV}^{2}$. From the measurement of the $W$ dependence of this process in eight bins of $t$, the $\rho^{0}$ Pomeron trajectory $\alpha_{\mathbb{P}}(t)$ has been extracted for the first time from the data of a single experiment. As shown in Fig.4a, the measurement is in good agreement with previous results and shows already a slope significantly smaller than the one of the soft Pomeron.

In the presence of a hard scale, like large values of $Q^{2}, t$, or the vector meson mass, perturbative QCD is expected to apply. Diffractive vector meson production can then be seen in the proton rest frame as a sequence of three subprocesses well separated in time: the fluctuation of the exchanged photon into a $q \bar{q}$ pair, the hard interaction of the $q \bar{q}$ pair with the proton via the exchange of (at least) two gluons in a color singlet state, and the $q \bar{q}$ pair recombination into a real vector meson. This approach results in a stronger rise of the cross section with $W$, which reflect the strong rise at small proton momentum fraction $x$ of the gluon density in the proton. Furthermore, to take into account the skewing effect, i.e. the difference between the proton momentum fractions carried by the two exchanged gluons, one has to consider Generalized Parton Distributions (GPDs) which extend the definition of standard PDFs by including additional information on the correlations between partons and their transverse motion.

$\mathrm{H} 1$ has presented comprehensive results [11] on elastic $J / \psi$ production in the $\gamma^{*} p$ center-of-mass energy ranges $40<W<$ $305 \mathrm{GeV}$ in photoproduction and $40<W<160 \mathrm{GeV}$ in DIS up to $80 \mathrm{GeV}^{2}$ in $Q^{2}$ and in both cases for $|t|<1.2 \mathrm{GeV}^{2}$. In such a process, the hard scale provided by the mass of the 

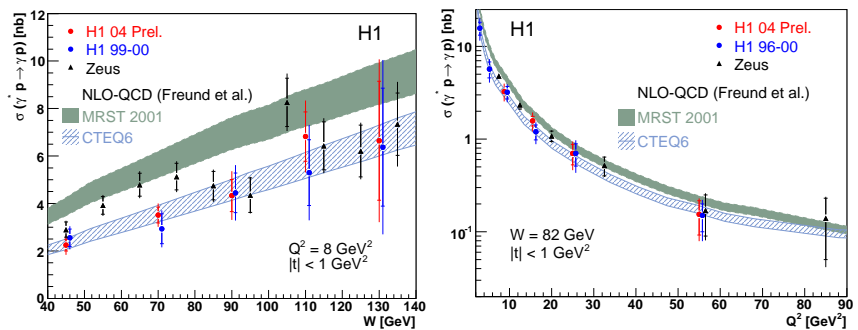

FIG. 5: New DVCS cross section as a function of $W$ and $Q^{2}$ as measured by H1. Superimposed are previous results from H1 and ZEUS as well as NLO calculations based on GPDFs.

involved charm quark ensures the validity of a perturbative QCD description. This is even more so in DIS where $Q^{2}$ can provide a second hard scale. The $Q^{2}$ and $W$ dependent $\gamma^{*} p$ cross-sections have been extracted. A steep rise with energy, $\sigma \propto W^{\delta}$, was observed with values of $\delta \simeq 0.75$ independently of $Q^{2}$. The effective Pomeron trajectories have been extracted from the study of the doubly differential $\mathrm{d} \sigma / \mathrm{dt}$ cross-section as a function of $W$ and $t$. In photoproduction, a positive value of $\alpha^{\prime}=0.164 \pm 0.028 \pm 0.030 \mathrm{GeV}^{-2}$ was obtained, leading to a shrinkage of the forward scattering peak, even if the effect is smaller than that observed in hadron-hadron interactions. In DIS, within its large error, the obtained value of $\alpha^{\prime}$ was found compatible both with the photoproduction result and with zero.

Photoproduction of vector mesons at large $|t|$ is largely studied since a few years as it is expected to be described by perturbative models involving the BFKL dynamics. These models predict a power law behaviour of the $t$ dependence of the cross section and a rise with $|t|$ of $\delta$ of the $W^{\delta}$ dependence. Proton-dissociative diffractive photoproduction of $J / \psi$ at high $t$ has been measured by ZEUS [12] and the cross section as a function of $W$ and $t$. The data for $|t|<M_{J / \psi}^{2}$ are well described by a model based on DGLAP evolution. The cross section rises significantly with increasing energy $W$ the higher $|t|$ the stronger the rise. This behaviour is reproduced by BFKL but not DGLAP as seen in Fig. 4b.

\section{DEEPLY VIRTUAL COMPTON SCATTERING}

The simplest process sensitive to GPDs is Deeply Virtual Compton Scattering (DVCS), i.e. the hard diffractive scatter- ing of a virtual photon off the proton $\gamma^{*} p \rightarrow \gamma p$ at small $|t|$ but large $Q^{2}$, which is fully calculable in perturbative QCD. A new high statistics analysis of DVCS has been performed by $\mathrm{H} 1$ [13] in the kinematic region $4<Q^{2}<80 \mathrm{GeV}^{2}$ using data from 2004. Fig. 5 shows the cross section as a function of $W$ and $Q^{2}$ together with previous measurements from $\mathrm{H} 1$ [14] and ZEUS [15]. The $W$ dependence can be parametrized as $\sigma \propto W^{\delta}$, yielding $\delta \cong 1.0$ at $Q^{2}=8 \mathrm{GeV}^{2}$, i.e. a value indicating the presence of a hard scattering. The DVCS cross section has been measured differentially in $t$ and the observed fast decrease with $|t|$ can be described by the form $e^{-b|t|}$ with $b=6.02 \pm 0.35 \pm 0.39 \mathrm{GeV}^{2}$ at $Q^{2}=8 \mathrm{GeV}^{2}$. This measurement allows to further constrain the models, as their normalization depends directly on the $t$ slope parameter. NLO QCD calculations using a GPD parametrization based on the ordinary parton distributions in the DGLAP region and where the skewedness is dynamically generated provide a good description of both the $Q^{2}$ and the $W$ dependences.

\section{CONCLUSIONS}

At HERA about $10 \%$ of the Deep Inelastic Scattering is diffractive. QCD fits have been performed and diffractive PDFs have been extracted. Measurements on exclusive diffractive processes are supporting the validity of QCD factorization in DIS. Results on diffractive dijet photoproduction disfavour factorization and leave room for theoretical and experimental improvements and speculations. A wide spectrum of processes and their dependence on the available hard scales have been studied and show that the properties of the Pomeron exchange at the larger scales are consistent with those of a hard process. Recent measurements on deeply virtual compton scattering and exclusive vector meson production from HERA are especially interesting because of their sensitivity to the Generalized Parton Densities. The rich physics program of HERA, aiming at a total of $500 \mathrm{pb}^{-1}$ by mid 2007, will help clarifying some of the ongoing issues and provide a useful input for the prediction of the diffractive exclusive cross sections at the LHC.

\section{Acknowledgements}

I would like to thank all the people involved in the conference preparation for the perfect organization and the warm atmosphere during the whole workshop.
[1] J. Collins, Phys. Rev. D 57, 3051 (1998) and erratum ibid. D 61, 019902 (2000).

[2] O. Gutsche, presented at ICHEP 2004, H1-prelim-04-113.

[3] M. Kapishin, presented at ICHEP 2006, ZEUS-prel-05-020.

[4] M. Kapishin, presented at ICHEP 2006, ZEUS-prel-05-001.

[5] H1 Collab., A. Aktas et al., preprint DESY 06-049, accepted by Eur. Phys. J.C.

[6] ZEUS Collab., S. Chekanov et. al., Eur. Phys. J. C 38, 43 (2004).

[7] CDF Collab., T. Affolder et al., Phys. Rev. Lett. 84, 5043 (2000).
[8] H1 Collab., A. Aktas et al., preprint DESY 06-164, submitted to Eur. Phys. J.C.

[9] ZEUS Collab., S. Chekanov et. al., Nucl. Phys. B 672, 3 (2003).

[10] J. Olsson, presented at DIS 2006, H1 prelim. 06-011.

[11] H1 Collab., A. Aktas et al., Eur. Phys. J. C 46, 585 (2006).

[12] K. Krüger, presented at ICHEP 2006, ZEUS-prel-05-006.

[13] B. Roland, presented at DIS 2006, H1-prel-06-012.

[14] H1 Collab., A. Aktas et al., Eur. Phys. J. C 44, 1 (2005).

[15] ZEUS Collab., S. Chekanov et al., Phys. Lett. B 573, 46 (2003) 